\title{
SUNSPOT MAGNETIC FIELDS AND HIGH ENERGY ELECTRONS
}

\author{
IN FLARES
}

\author{
TATSUO TAKAKURA
}

Tokyo Astronomical Observatory, University of Tokyo, Mitaka, Tokyo, Japan

\begin{abstract}
A balloon observation of an impulsive hard X-ray burst on September 27, 1969 showed the size of the source to be one arc minute or less. It was remarkably smaller than the associated $\mathrm{H} \alpha$ flare with a size of 3 arc min.

The efficient acceleration of electrons and the trigger of the flares are suggested to be attributed to a large scale electric potential field caused by a gas motion near the photosphere. The primary cause of the onset of flares would be the acceleration of electrons. The electrons excite plasma waves which make the conductivity lower by several orders, so that the electromagnetic energy $I^{2} L$ stored before the onset of the flare would be suddenly converted into the heat due to the ohmic loss.
\end{abstract}

At the early phase of flares, electrons are accelerated up to about $1 \mathrm{MeV}$ in the sunspot magnetic field in the short time of 10 to $100 \mathrm{~s}$. The total number of the electrons accelerated above $50 \mathrm{keV}$ is estimated to be $10^{35}-10^{36}$. The behavior of the energetic electrons in a flare region has been investigated by using hard X-ray bursts and microwave radio bursts.

The present paper will briefly show the results of a recent balloon observation of the location and size of the source of an impulsive hard X-ray burst, and propose a model to generate an electric potential field, which accelerates electrons to emit hard X-ray and microwave impulsive bursts and also to be a trigger for the flare.

\section{Balloon Observation of the Position and Size of a Hard X-Ray Burst}

On September 27, 1969 a balloon observation of the position and size of a hard X-ray burst was made successfully in cooperation with the Institute of Space and Aeronautical Science (Takakura et al., 1971). A one dimensional modulation collimator with a half-power width of 1.3 arc min was used (Figure 1). The observed energy range was effectively $30-60 \mathrm{keV}$.

A flare of importance 3 occurred accompanied by a comparatively small hard $\mathrm{X}$-ray impulsive burst and a microwave burst.

The center of the X-ray source was on the line passing through the center of a big $\mathrm{H} \alpha$ flare region of 3 arc min diameter (Figure 2). The size of the X-ray source was 1 arc min or less which is remarkably smaller than the $\mathrm{H} \alpha$ flare (Figure 3).

On the other hand, the position and size of the source of the associated microwave burst were observed at Toyokawa with 1-dimensional interferometers (Tanaka and Énomé, 1971). The radio position is also on a line passing through the center of the flare region although the scanning direction was different by $27^{\circ}$ from that of the $\mathrm{X}$-ray collimator as shown in Figure 2 . The measurement of the radio source size was not accurate enough (less than $1.8^{\prime}$ ) in this event to judge whether the sources of the $\mathrm{X}$-rays and the microwaves are coincident or not. 
The observed size of 1 arc min or less of the hard X-ray source is comparable with or smaller than the source size of normal microwave impulsive bursts so far observed at Toyokawa. On the other hand, there is an excess of a factor $10^{2}$ in the total number of electrons emitting the hard X-ray bursts compared with the electrons emitting the concurrent microwave bursts (Takakura, 1969; Takakura and Scalise, 1970). This implies that the acceleration of a majority of the electrons is made in a small region in which the gyro-synchrotron emission of radio waves is negligible but hard X-rays

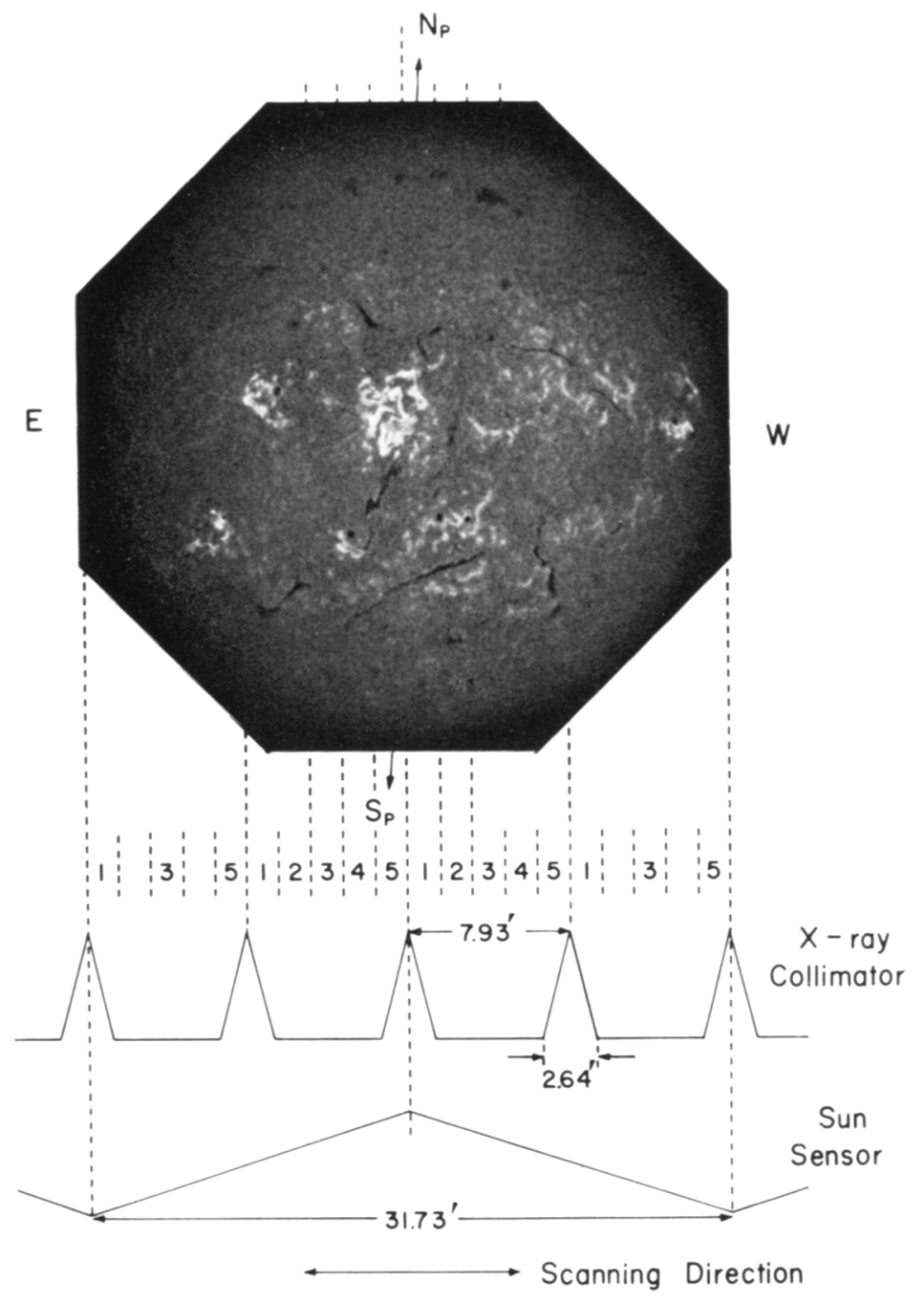

Fig. 1. One-dimensional transmission responses of the X-ray modulation collimator and the Sunsenor (lower curves). The $\mathrm{H} \alpha$ photograph of the hard X-ray burst, $03^{\mathrm{h}} 57^{\mathrm{m}} 06^{\mathrm{s}} \mathrm{UT}$ on September 27 , 1969 is available by courtesy of the Carnarvon Tracking Station and the World Data Center A, ESSA, Boulder. 
are predominant. This condition is satisfied in a plasma with a density of $10^{10} \mathrm{~cm}^{-3}$, if the magnetic field is less than $30 \mathrm{G}$, or if the magnetic field is less than $100 \mathrm{G}$ and the energies of the electrons are less than $1 \mathrm{MeV}$. In these cases low frequencies are suppressed by the Razin effect (Ramaty, 1969), while the emissivity at the higher frequencies is low due to the weak magnetic field. The microwave impulsive burst

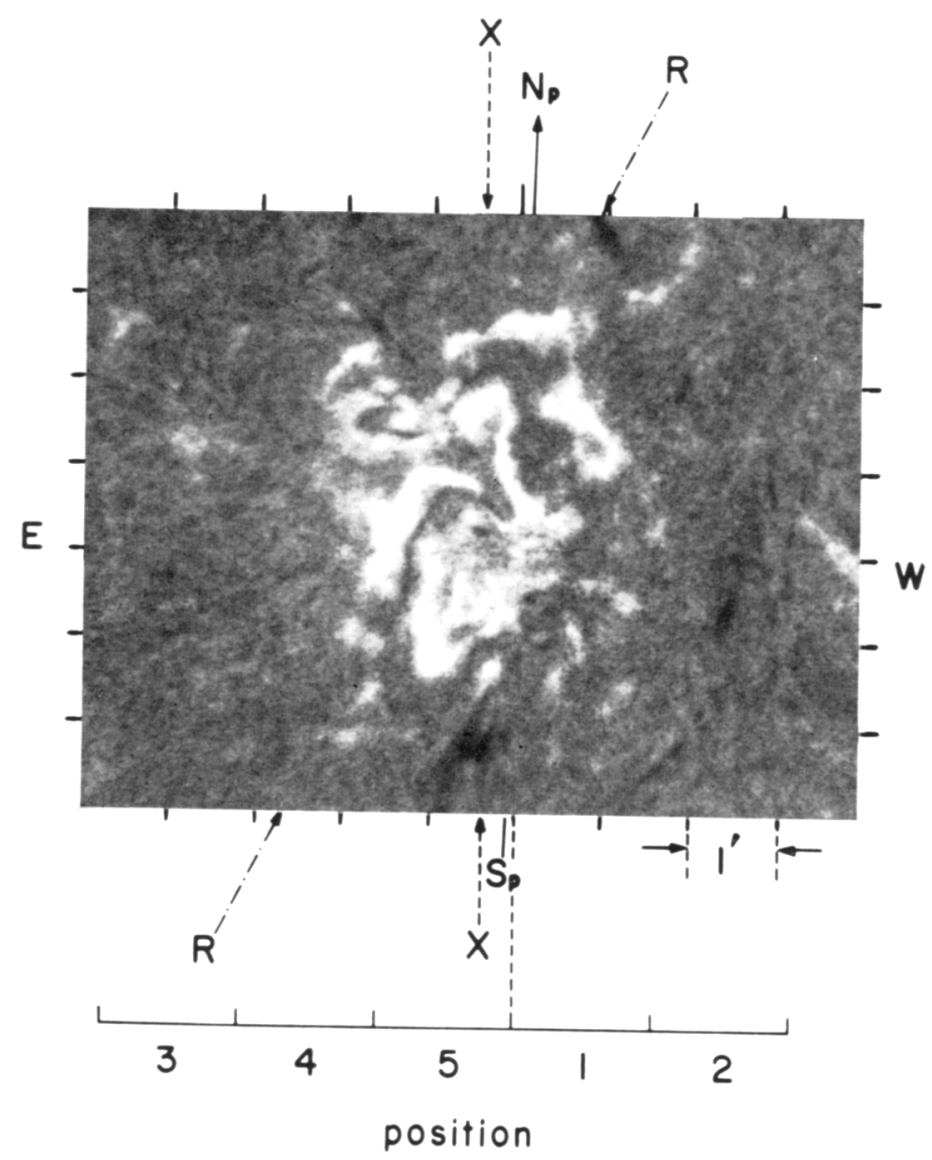

Fig. 2. The $\mathrm{H} \alpha$ flare region (cf. Figure 1) and the location of the source of the hard X-ray burst. The center of the X-ray sources was located on the line X. The center of the source of associated radio burst at $3750 \mathrm{MHz}$ was located on the line $R$.

may be emitted from a much smaller number of electrons diffused into the regions with higher magnetic fields or accelerated there.

In order to accelerate $10^{36}$ electrons above $50 \mathrm{keV}$ up to $1 \mathrm{MeV}$ in 10 to $100 \mathrm{~s}$ in a region of the order of $10^{4} \mathrm{~km}$, the efficiency of acceleration must be very high. Therefore, I would suggest the following process for the generation of large scale electric potential fields to accelerate electrons and to trigger the flares. 


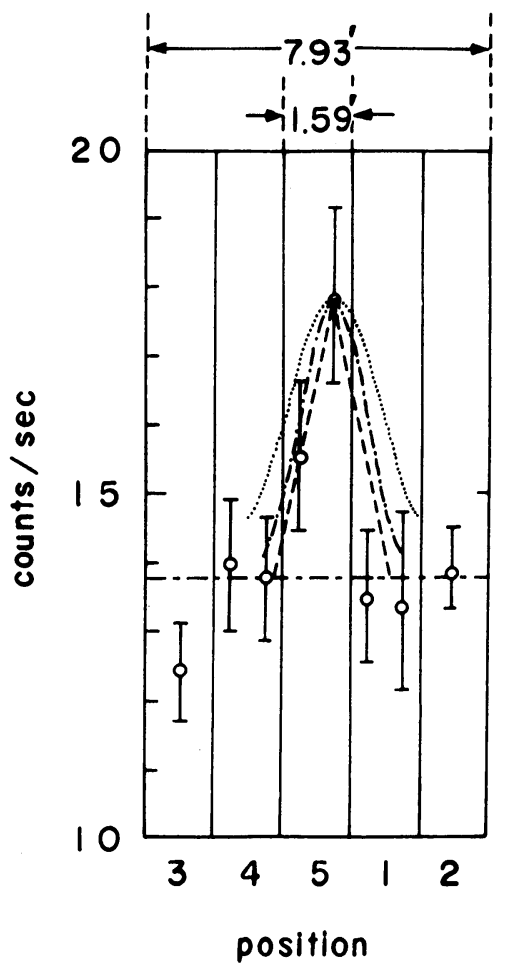

Fig. 3. One-dimensional intensity distribution of the hard X-rays during the burst from 0356 to 0358.5 UT. Position numbers correspond to those given in Figures 1 and 2. Dashed curve: expected profile for a point source. Dot-dashed curve: expected profile for a gaussian source with a half power width of 1.1 arc min. Dotted curve: gaussian source of 2.2 arc min.

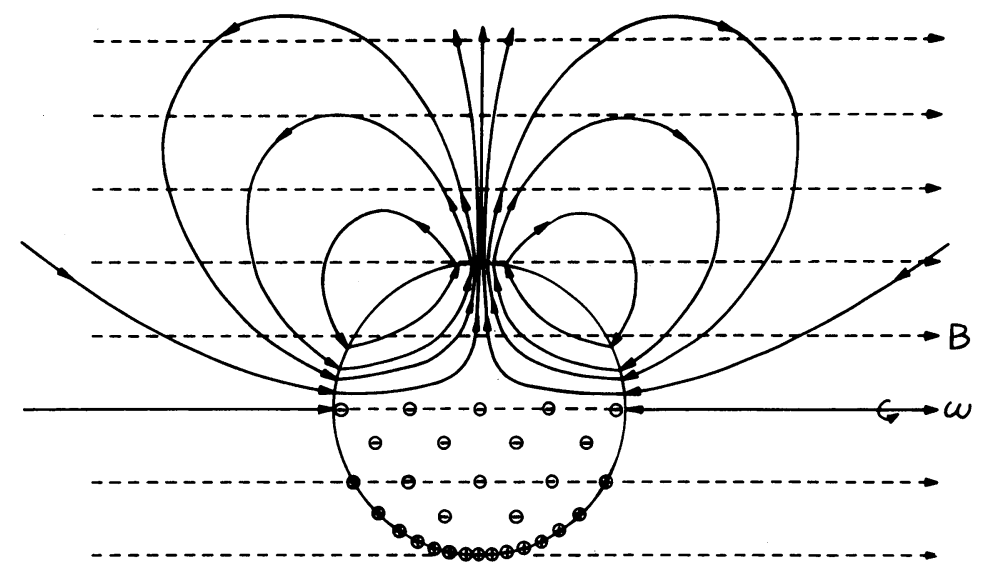

Fig. 4. The electric lines of force for $\mathbf{E}_{0}$ and $\mathbf{E}_{i}$ (effective). They also show the paths of the steady current. The steady charge distribution is shown schematically in the lower half. 


\section{Generation of Potential Electric Fields by a Rotating Gas Cloud in a Magnetic Field}

Suppose that a gas cloud rotates in a uniform magnetic field near the photosphere, where the gas pressure is greater than the magnetic pressure, and the conductivity is nearly isotropic due to the fact that the collision frequency is higher than the gyrofrequency.

In the rotating cloud, a Lorentz force $q(\mathbf{v} \times \mathbf{B})$ produces a charge separation. The charge distributions in the cloud and at its surface produce potential electric fields not only in the cloud but also outside the cloud. Since the outside of the cloud is also a conducting gas, a current flows from the outside through the rotating cloud.

In the steady state, the following quadrupole potential field and a steady current can be obtained. It is assumed that the conductivity $\sigma$ is isotropic and uniform, and the rotation axis is pafallel to a uniform magnetic field.

In spherical coordinates $(r, \theta, \varphi)$ and in MKS units, we have the following potential field,

$$
\begin{aligned}
r \geqslant a: \quad V_{0} & =\frac{B \omega a^{2}}{15}\left(\frac{a}{r}\right)^{3}\left(1-3 \cos ^{2} \theta\right), \\
\mathbf{E}_{0} & =-\operatorname{grad} V_{0} \\
& =\frac{B \omega a}{5}\left(\frac{a}{r}\right)^{4}\left\{\left(1-3 \cos ^{2} \theta\right) \mathbf{e}_{r}-2(\sin \theta \cos \theta) \mathbf{e}_{\theta}\right\}, \\
r \leqslant a: \quad V_{i} & =B \omega a^{2}\left\{-\frac{1}{3}+\frac{1}{5}\left(\frac{r}{a}\right)^{2}\left(2-\cos ^{2} \theta\right)\right\}, \\
\mathbf{E}_{i}(\text { effective }) & =\omega \times \mathbf{r} \times \mathbf{B}-\operatorname{grad} V_{i} \\
& =\frac{B \omega a}{5}\left(\frac{r}{a}\right)\left\{\left(1-3 \cos ^{2} \theta\right) \mathbf{e}_{r}+3(\sin \theta \cos \theta) \mathbf{e}_{\theta}\right\},
\end{aligned}
$$

where $\omega$ is the angular frequency of the rotating gas-sphere with a radius $a$. (Incidently, if the outside of the rotating sphere is a vacuum, $\mathbf{E}_{0}$ is $\frac{5}{2}$ times the above value and $E_{i}($ eff. $)=0$, e.g., Davis, 1947; Backus, 1956).

The steady current is given by $\mathbf{J}_{0}=\sigma \mathbf{E}_{0}$ and $\mathbf{J}_{i}=\sigma \mathbf{E}_{i}$ (effective), and $\operatorname{div} \mathbf{J}=-\partial \varrho / \partial t=0$ is satisfied. The paths of the steady current are illustrated in Figure 4. They also indicate the lines of force of $\mathbf{E}_{0}$ and $\mathbf{E}_{i}$ (effective). The external electronic field $\mathbf{E}_{0}$ has generally a component parallel to the magnetic field. A part of the electric field penetrates through the corona, in which the conductivity is anisotropic due to the smaller collision frequency, so that the electric field would be modified, and also the current flows almost along the magnetic field.

The electric field strength is of the order of $0.3 B \omega a$ near the surface $(r=a)$ of the rotating gas, and the maximum potential difference is $0.2 B \omega a^{2}$. If we take $\omega a=4 \mathrm{~ms}^{-1}$ and $B=0.3 \mathrm{Wbm}^{-2}(3000 \mathrm{G})$, we have $E_{\max } \simeq 10^{2.5} \mathrm{Vkm}^{-1}$ and $V_{\max } \simeq 10^{6.5} \mathrm{~V}$ for $a=10^{4} \mathrm{~km}$. Therefore, it would be possible for the electric field along the magnetic field in the lower corona to be $1-10 \mathrm{Vkm}^{-1}$ or more.

The time scale to come to a steady state would be long. However, due to a skin 
effect, any rapid variation in the electric field would be followed by a current, although the current is confined in thin layers or thin pipes or threads. The thickness is given by the skin depth $\delta \simeq \sqrt{D \tau}$, where $D$ is the diffusion coefficient and $\tau$ is the time scale for the variation of the electric field $\left(D=1 / \sigma \mu_{0} \simeq 10^{6} \mathrm{~m}^{2} / \mathrm{s}\right.$ at the photosphere). It may be noted that the frozen condition is not necessarily satisfied if the skin depth is reasonably taken as the characteristic length.

Before the onset of the flare, a current may be flowing in the lower corona due to an electric field of a few $\mathrm{V} / \mathrm{km}$. The gas motion which deforms the sunspot configuration or differential rotation would generate the electric field. If the current is $I$, and an equivalent inductance of the circuit is $L$, the energy $I^{2} L$ is stored. The $L$ is equivalent to the magnetic field created by $I$. Both the current and the inductance would not be in a steady state as Alfvén and Carlqvist $(1967,1969)$ have suggested but would increase with time according to an increase in the skin depth. The $L$ and $I$ are estimated to be $10^{-3}$ Henry and $10^{14} \mathrm{~A}$, respectively, for a time scale of one day and for an

\section{TABLE I}

Summary of the flare model

\begin{tabular}{|c|c|c|}
\hline & Energy storage & Trigger \\
\hline $\begin{array}{l}\text { Gas motion near } \\
\text { the photosphere }\end{array}$ & \multirow[t]{2}{*}{$\begin{array}{l}\text { Gradual } \\
\tau \simeq \text { day }\end{array}$} & $\begin{array}{l}\text { Impulsive } \\
\tau \simeq 10-100 \mathrm{~s}\end{array}$ \\
\hline $\begin{array}{c}\downarrow \\
\text { Charge distribution in } \\
\text { the moving gas } \\
\downarrow\end{array}$ & & \\
\hline \multirow[t]{5}{*}{$\begin{array}{l}\text { Electric field outside } \\
\text { and inside }\end{array}$} & $\begin{array}{l}E \simeq 5 \mathrm{~V} / \mathrm{km} \\
\text { in the lower corona }\end{array}$ & $\begin{array}{l}E \geqslant 15 \mathrm{~V} / \mathrm{km} \\
\text { in the lower corona }\end{array}$ \\
\hline & $\begin{array}{l}\downarrow \\
I \text { and } L \text { (both are } \\
\text { functions of } \tau \text { ) }\end{array}$ & $\begin{array}{l}\downarrow \\
\text { Acceleration of electrons } \\
(K \leqslant 1 \mathrm{MeV})\end{array}$ \\
\hline & $\stackrel{\downarrow}{\downarrow}$ Stored energy $I^{2} L$ & $\begin{array}{c}\downarrow \\
\text { Electron plasma waves } \\
\downarrow \\
\text { Decrease in conductivity }\end{array}$ \\
\hline & $\begin{array}{l}\downarrow \\
\text { Energy release due to ohmic loss } \\
(\tau \simeq 1-10 \mathrm{~s}) \\
\text { Flare }\end{array}$ & $\begin{array}{l}\downarrow \\
\text { Hard X-ray burst } \\
\text { Microwave impulsive burst } \\
\text { Type III bursts }\end{array}$ \\
\hline & $\begin{array}{l}\text { Sporadic coronal condensation } \\
\text { Gas ejection } \\
\text { M.H.D. waves } \\
\quad \downarrow \\
\text { Acceleration of protons and } \\
\text { electrons to higher energies }\end{array}$ & \\
\hline
\end{tabular}


electric field strength of $5 \mathrm{~V} / \mathrm{km}$ in the lower corona. In this case the stored energy is $10^{25}$ joules which is required for a big flare.

Now, if the electric field exceeds about $10 \mathrm{~V} / \mathrm{km}$ in the lower corona due to an additional faster impulsive gas motion near the photosphere, almost all of the thermal electrons are accelerated to higher energies, since the acceleration rate exceeds the collisional loss rate. This would be a critical condition for the trigger of flares. In this electric field one second is enough to accelerate electrons up to relativistic energies. However, in order to accelerate $10^{36}$ electrons above $50 \mathrm{keV}, 10^{2} \mathrm{~s}$ may be required in order to increase the skin depth.

The accelerated electrons excite electron plasma waves due to the two beam instability, and the waves spread over the regions in which $I$ is flowing. The plasma waves decrease the conductivity by 5 orders in the corona (Buneman, 1959; Hamberger and Friedman, 1968). Accordingly, the stored energy $I^{2} L$ would be converted suddenly into the thermal energy due to the ohmic loss. This time scale $L / R$ is estimated to be $1-10 \mathrm{~s}$.

The accelerated electrons also emit hard X-rays, and a part of the electrons diffuse into the stronger field region to emit a microwave impulsive burst.

More details will be published elsewhere.

\section{Summary}

A summary of the flare model is shown in Table $I$.

\section{References}

Alfvén, H. and Carlquist, P.: 1967, Solar Phys. 1, 220.

Backus, G.: 1956, Astrophys. J. 124, 508.

Buneman, O.: 1959, Phys. Rev. 115, 503.

Carlqvist, P.: 1969, Solar Phys. 7, 377.

Davis, L., Jr.: 1947, Phys. Rev. 72, 632.

Hamberger, S. M. and Friedman, M.: 1968, Phys. Rev. Letters 21, 674.

Ramaty, R.: 1969, Astrophys. J. 158, 753.

Takakura, T.: 1969, Solar Phys. 6, 133.

Takakura, T. and Scalise, E., Jr.: 1970, Solar Phys. 11, 434.

Takakura, T., Ohki, K., Shibuya, N., Fujii, M., Matsuoka, M., Miyamoto, S., Nichimura, J., Oda, M., Ogawara, Y., and Ota, S.: 1971, Solar Phys. $16,454$.

Tanaka, H. and Énomé, S.: 1971, Solar Phys. 17, 408.

\section{Discussion}

Cowling: Could the problem of flare energies have been solved along these lines, it would have been solved 30 yr ago. Takakura has ignored induction effects corresponding to changes $\partial B / \partial t$. I conservatively estimate that these may reduce the efficiency of his mechanism in the ratio $10^{-10}$.

Takakura: I have obtained the solution in the steady state at which $\partial B / \partial t=0$. The time to be steady would be long. However, as I have suggested, if we take the 'skin effect' into account any rapid variation of the electric field is followed by the current, though the current would be concentrated in thin filaments or thin pipes. This effect has been taken into account in my numerical estimates for the flare energy and for the trigger. Furthermore, if we take the skin depth as a characteristic length, the frozen condition is not necessarily satisfied near the photosphere. 